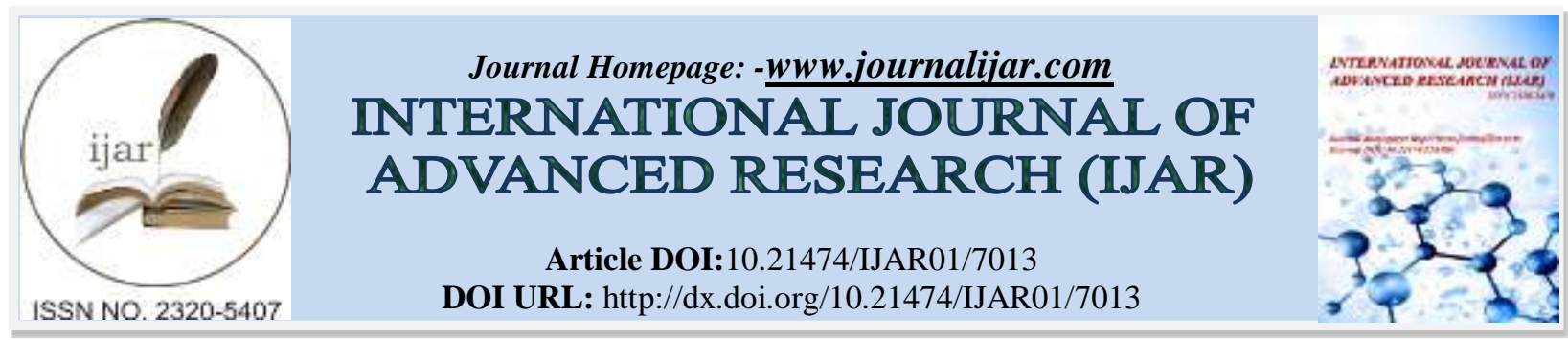

RESEARCH ARTICLE

\title{
ANTIENCEPHALITIC ACTIVITIES OF BIOACTIVE SUBSTANCES PARTIALLY PURIFIED FROM LIRIOPE PLATYPHYLLA TUBER.
}

\author{
Woo Young Bang ${ }^{1}$, Chi Won Noh ${ }^{2}$, Seung-Ho Jeon ${ }^{3}$, Yeon Jo Ha ${ }^{4}$, Jeong Mi Kang ${ }^{4}$, Chae Won Lee ${ }^{1}$, Il-Suk \\ $\mathrm{Kim}^{4}$, Tae Wan Kim ${ }^{4}$ and Sam Woong Kim ${ }^{4}$. \\ 1. National Institute of Biological Resources (NIBR), Environmental Research Complex, Incheon 22689, South \\ Korea. \\ 2. Gyeongsangnam-do Agricultural Research \& Extension Services, Jinju, 52733, South Korea. \\ 3. Department of Agronomy \& Medicinal Plant Resources, Gyeongnam National University of Science and \\ Technology, Jinju, 52725, South Korea. \\ 4. Swine Science and Technology Center, Gyeongnam National University of Science \& Technology, Jinju 52725, \\ South Korea.
}

\section{Manuscript Info}

Manuscript History

Received: 01 March 2018

Final Accepted: 03 April 2018

Published: May 2018

Keywords:-

Antiencephalitis, bioactive substance, $l$.

Platyphylla, fraction, eluent, sep-pak r18.

\begin{abstract}
The Goal of this study was to identify whether the extract of Liriope platyphylla tuber can induce antiencephalitis. The yield of the hot water extraction was 1.5 times higher than the extract by simple homogenization. When the methanol fractionation after the hot water extract was done, the precipitated pellet in $70 \%$ methanol showed the highest antiencephalitis activity. In organic solvent partition after $70 \%$ methanol extract, the water phases in hexane, chloroform and ethyl acetate were exhibited higher activities, whereas its activity in butanol partition was divided into water and butanol layers. The eluents from the Sep-Pak R18 cartridge by fraction derived from the butanol and water phases in butanol partition showed the highest activity in the fraction of the hexane: ethyl acetate (5: 5) and ethyl acetate, respectively. It is assumed that the antiencephalitic activity is maintained not only in the hydrophilic materials but also in the hydrophobic ones. Therefore, we suggest that specific metabolites retained in L. platyphylla tuber are a high possibility to be applied as antiencephalitic agents.

Copy Right, IJAR, 2018,. All rights reserved.
\end{abstract}

\section{Introduction:-}

Until recently, L. platyphylla tuber has been used as a raw material for herbal medicines rather than as a sole medicinal herb. Therefore, although the behavior of consumption is not high, recently, as the interest of consumers in health functional foods has increased, the tuber has been produced as functional foods for various health aids. As a result, the consumption of $L$. platyphylla tuber has been also increased, but the direct consumption of $L$. platyphylla tuber seems owing to the fact to be changed a little in the consumption pattern of the tuber. This has been derived by progress continuously from the research for the functional effect and cultivation of L. platyphylla tuber. In addition, the perception of consumption of $L$. platyphylla tuber is not fixed in the viewpoint of raw materials of herbal medicines, but changed towards application in food. 
In South Korea, L. platyphylla Wang et Tang, which is widely grown in the southern region, is mainly used as a medicine, and referred to as Korean Liriope platyphylla (Kim et al., 2001; Shin, 2002). The efficacy of L. platyphylla is known to have nourishment, tussive, mucoactive and diuretic effects as a blood sugar reduction and a nourishing agents. In order to obtain such an effect, it is used in various herbal medicines (Han DS 1993; Shibata et al., 1971). L. platyphylla is divided into Korean Liriope platyphylla Wang et Tang, Liriope spicata L. and Ophiopogon japonicas K. G. L. platyphylla tuber maintains as the main active ingredients of steroidal saponins such as spicatoside and ophipogonin, as well as produces various polysaccharides such as $\beta$-sitosterol, stigmasterol, $\beta$ sitosterol glucoside and oligosaccharides (Back et al., 1998; Shin, 2002). Among them, steroid saponin has been reported to have anti-cancer effects (Choe and Yang,1982). Especially, ophiopogonin D and spicatoside A among various components of L. platyphylla are well-known for antioxidant, anti-inflammatory and neutrophic effects (Qian et al., 2010; Kou et al., 2005; Hur et al., 2009).

In this study, we analyzed the possibility of application to healthy functional foods via examination of antiencephalitis according to hot water extract, organic solvent fractionations, and column purification of $L$. platyphylla.

\section{Materials and Methods:-}

\section{Extractions of oriental medicinal herbs:-}

Herbal medicines were purchased in a traditional market in South Korea. The herbal medicines were mixed with each solvent at a ratio of 1: 9, and then extracted according to the conditions of hot water, cold water, methanol, and ethanol. The hot water extraction was carried out by 3 times at $100^{\circ} \mathrm{C}$ for 3 hours, the extracts were combined, concentrated to 4 times, and then lyophilized. The cold water extract was left at room temperature for 24 hours, extracted, concentrated and then lyophilized. Ethanol and methanol were left at $4^{\circ} \mathrm{C}$ for 24 hours at each treatment concentration, extracted, concentrated and then lyophilized. The dried extracts from the herbal medicines were used as samples for study.

The purchased L. platyphylla tuber was mixed with distilled water at a ratio of 1: 9, homogenized at 13,500 rpm, centrifuged at 2,000 rpm for 20 minutes, the supernatant was collected and then concentrated by 4 times. On the other hand, a sample of L. platyphylla tuber, extracted three times at $100^{\circ} \mathrm{C}$ for 90 minutes after suspension with the same mixing ratio, the extracts were combined and then concentrated by 4 folds. The concentrated extracts from L. platyphylla tuber were used as samples for study.

\section{methanol fractionation:-}

The concentrated extracts from L. platyphylla tuber were introduced for methanol fractionation with 30,50 and $70 \%$ methanol. After adjusting for each concentration of methanol, the reaction was carried out at $4^{\circ} \mathrm{C}$ for 1 hour and centrifugation was carried out at 2,000 rpm for 20 minutes to separate the supernatant and the precipitate. The supernatant was concentrated 10 times with respect to the amount of the original amount, and the precipitate was resuspended in distilled water of the same amount as the supernatant,

\section{Organic solvent partition:-}

The $70 \%$ methanol precipitate was mixed with the same amount of butanol, ethyl acetate, chloroform and hexane. The mixed samples were incubated at $4^{\circ} \mathrm{C}$ for 1 hour and then separated by centrifugation at 2,000 rpm for 20 minutes. Each separated layer was collected, concentrated for removing organic solvents and resuspended in distilled water of the same amount as the original volume.

\section{Sep-Pak cartridge purification:-}

The extracts in the butanol and water layers of butanol partition was loaded on a Sep-Pak ${ }^{\circledR}$ plus C18 cartridge (Waters). The loaded sample were eluted with $20 \mathrm{ml}$ of each solution of hexane, hexane: ethyl acetate (8: 2, 6: 4, 5: 5, 4: 6 and 2: 8) and ethyl acetate (saturated by water/methanol). The eluted extract was concentrated to remove the organic solvent and resuspended in water to a 10-fold concentration.

\section{Microglial cells (BV-2) culture:-}

The microglial cells used in this study were done by BV-2 cell line isolated from mouse brain tissue and supported by Myung-sook Oh of Kyung Hee University. The medium for BV-2 cell culture was DMEM-low glucose medium (Sigma-Aldrich Korea, Seoul, South Korea), glucose $4 \mathrm{~g} / \mathrm{l}$, sodium bicarbonate $3.7 \mathrm{~g} / \mathrm{l}, 10 \%$ fetal bovine serum (FBS) 100 units/ml penicillin and $100 \mu \mathrm{g} / \mathrm{ml}$ streptomycin), the final $\mathrm{pH}$ was adjusted to $7.2-7.4$, and then sterilization was 
performed using a filter having a pore size of $0.22 \mu \mathrm{m}$. Since the supported BV-2 cells contained toxic DMSO, the DMSO was removed through a washing procedure and then cultured at $37^{\circ} \mathrm{C}$ under $5 \% \mathrm{CO}_{2}$ using the medium.

\section{Sample pretreatment and induction of encephalitis reaction of microglia (BV-2):-}

In this study, samples were pretreated with BV-2 cells prior to the induction of encephalitis in order to examine the preventive effects of the samples to be analyzed. For this purpose, the DMSO concentration used to dissolve the sample to be analyzed was treated with the final $0.5 \%$ and used as a negative (-) control. After pretreatment of the assay samples, lipopolysaccharide (LPS; Sigma-Aldrich Korea, Seoul, South Korea) was used to induce encephalitis responses in the BV-2 cell line. Thus, BV-2 cell culture medium was treated with LPS at a concentration of $1 \mu \mathrm{g} / \mathrm{ml}$ and reacted for 19 hours or 24 hours.

Antiencephalitic assay in encephalitis-induced microglia (BV-2):-

BV-2 cells were seeded into each well of a 96-well plate at $0.5 \times 10^{5}$ cells, and the cells were used for analysis after fixation on the bottom of each well. Cytotoxicity was assessed using the MTS assay kit (Promega, Madison, Wis., USA) after pretreatment for 5 hours and LPS treatment for 19 or 24 hours. As MTS assay, BV-2 cells were seeded in each well of a 96-well plate, and when the sample to be analyzed was pretreated for 5 hours and treated for 19 hours or 24 hours, the supernatant of the medium was measured for nitric oxide. In order to compare the amount of nitric oxide secreted by BV-2 cells induced by encephalitis, the obtained medium samples were mixed with Griess reagent (Enzo Life Sciences, Inc., Farmingdale, NY, USA) at a ratio of 1: 1, and then OD value at $540 \mathrm{~nm}$ was measured using a Microplate reader (Synergy HT).

\section{Statistical analysis:-}

The collected data were analyzed by GLM (General linear model) of SAS program (V. 9.2, Cary, NC, USA). Mean values was verified by 5\% significant level in Duncan's multiple range test (DMRT).

\section{Figure legends:-}

Fig. 1:-Antiencephalitic activities of crude extracts from oriental medicines.

The left and right panels indicate NO and MTS assays, respectively. The test cell was used by microglial BV2. The concentrates of the crude medicinal extracts were applied by $300 \mathrm{ug} / \mathrm{ul}$. C1: normal cell, C2: LPS treated, a;hot water extract of L. platyphylla, b; $70 \%$ ethanol extract of L. platyphylla, c; $100 \%$ methanol extract of C. Rhizoma, d; $100 \%$ methanol extract of $R$. coreanus, e; hot water extract of $P$. notoginsengs, f; $100 \%$ methanol extract of $P$. notoginsengs, $\mathrm{g} ; 100 \%$ methanol extract of $C$. sappan, h: cold water extract of $C$. sappan, i: $70 \%$ ethanol extract of C. cassia Presl., j: 100\% methanol extract of C. cassia Presl.

Fig. 2:-Antiencephalitic activities according to methanol precipitation of $L$. platyphylla extracts.

The crude extracts were performed by hot water extractions. Methanol concentrations were adjusted into 30, 50 and $70 \%$. The solutions were separated by centrifuge. The supernatants and precipitated pellets were suspended into distilled water. The test cell was used by microglial BV2. C1; non-treated, C2 WP70; treated with LPS (1 ug/ml) for $24 \mathrm{hr}, \mathrm{H}$; homogenized for $1 \mathrm{~min}$ at 13,000 rpm and then directly extracted, W; after homogenized for $1 \mathrm{~min}$ at $13,000 \mathrm{rpm}$, and then extracted by 3 times for $90 \mathrm{~min}$ at $100^{\circ} \mathrm{C}$. 30, 50 and 70 ; final methanol concentrates, S; supernatant of centrifugation for $20 \mathrm{~min}$ at $2,000 \mathrm{rpm}$, P; re-suspended solution to the same volume with the supernatant for pellet of centrifugation for $20 \mathrm{~min}$ at $2,000 \mathrm{rpm}$,

Fig. 3:-Results of antiencephalitic activities by organic solvent partition production.

$70 \%$ methanol precipitated fraction was treated by each solvent. The treated solutions were separated by centrifugal force and then concentrated by an evaporator.

Fig. 4:-Antiencephalitic activities of eluents from Sep-Pak R18 catridge loaded by butanol phase in butanol partition.:-

Each eluent was eluted by the solvents as described below. The eluents were concentrated by an evaporator. B.FT; flow through from butanol phase in butanol partition, B.Hex Et 8:2, 6:4, 5:5, and 2:8; eluents by hexane:ethyal acetate (8:2, 6:4, 5:5, and 2:8) from butanol phase, B·Hex; eluent by hexane from butanol phase, B.Et (W/M); eluent by ethyl acetate saturated with water and methanol from butanol phase. 
Fig. 5:-Antiencephalitic activities of eluents from Sep-Pak catridge loaded by water phase in butanol partition.

Each eluent was eluted by the solvents as described below. The eluents were concentrated by an evaporator. B.FT; flow through from water phase in butanol partition, B·Hex Et 8:2, 6:4, 5:5, and 2:8; eluents by hexane:ethyal acetate $(8: 2,6: 4,5: 5$, and 2:8) from water phase, B·Hex; eluent by hexane from water phase, B.Et (W/M); eluent by ethyl acetate saturated with water and methanol from water phase.

Table 1:-Yield according to extraction method

\begin{tabular}{|l|c|}
\hline \multicolumn{1}{c|}{ Extraction method } & Yield (\%) \\
\hline Homogenization & 48.8 \\
\hline Hot water & 74.7 \\
\hline
\end{tabular}

Fig. 1:-
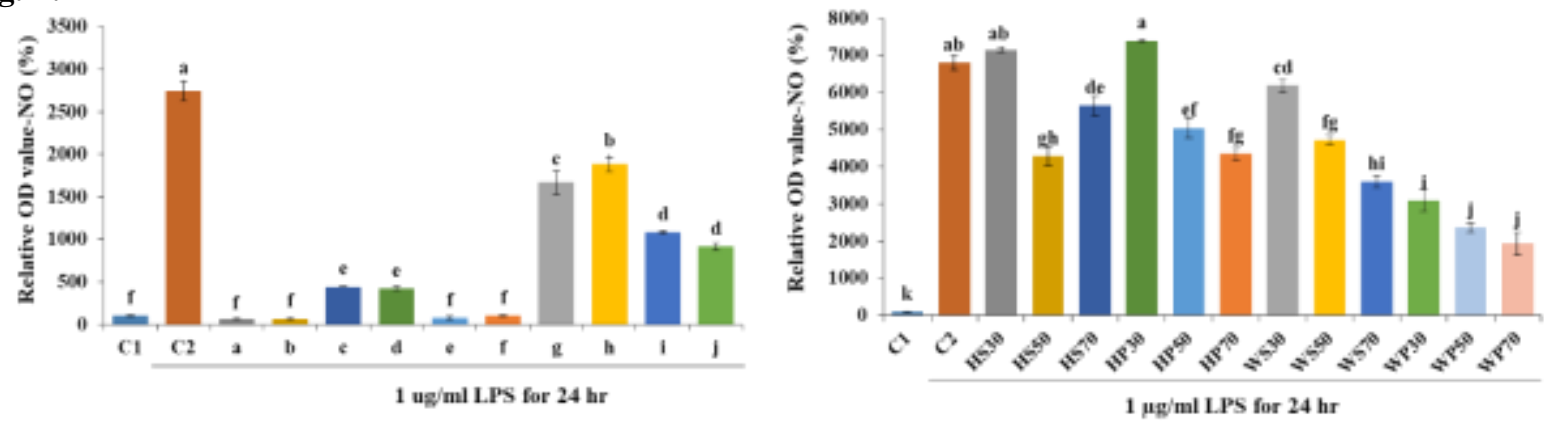

Fig 2:-

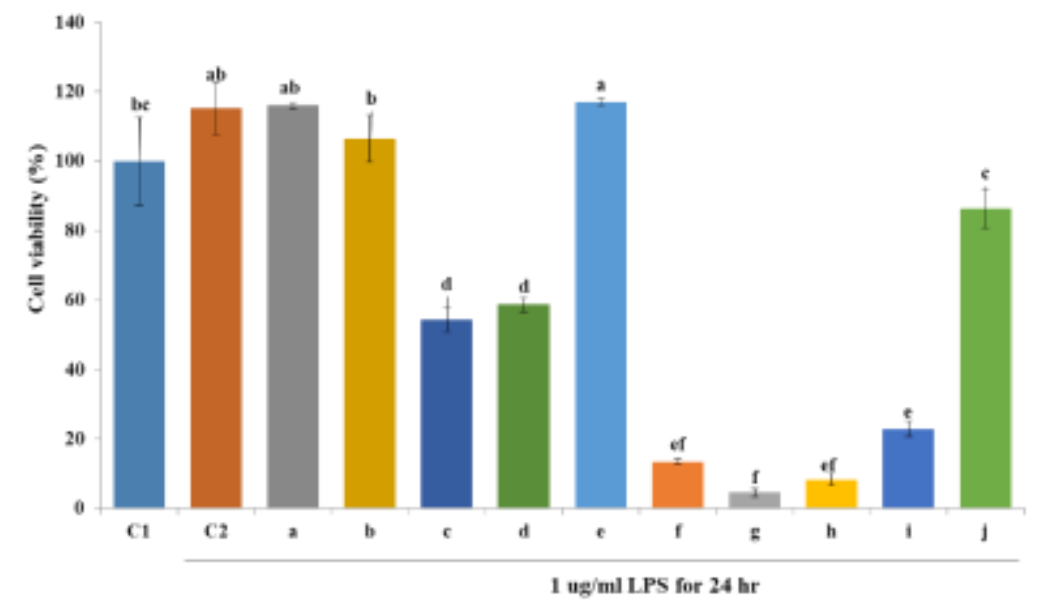

\section{Fig 3:-}

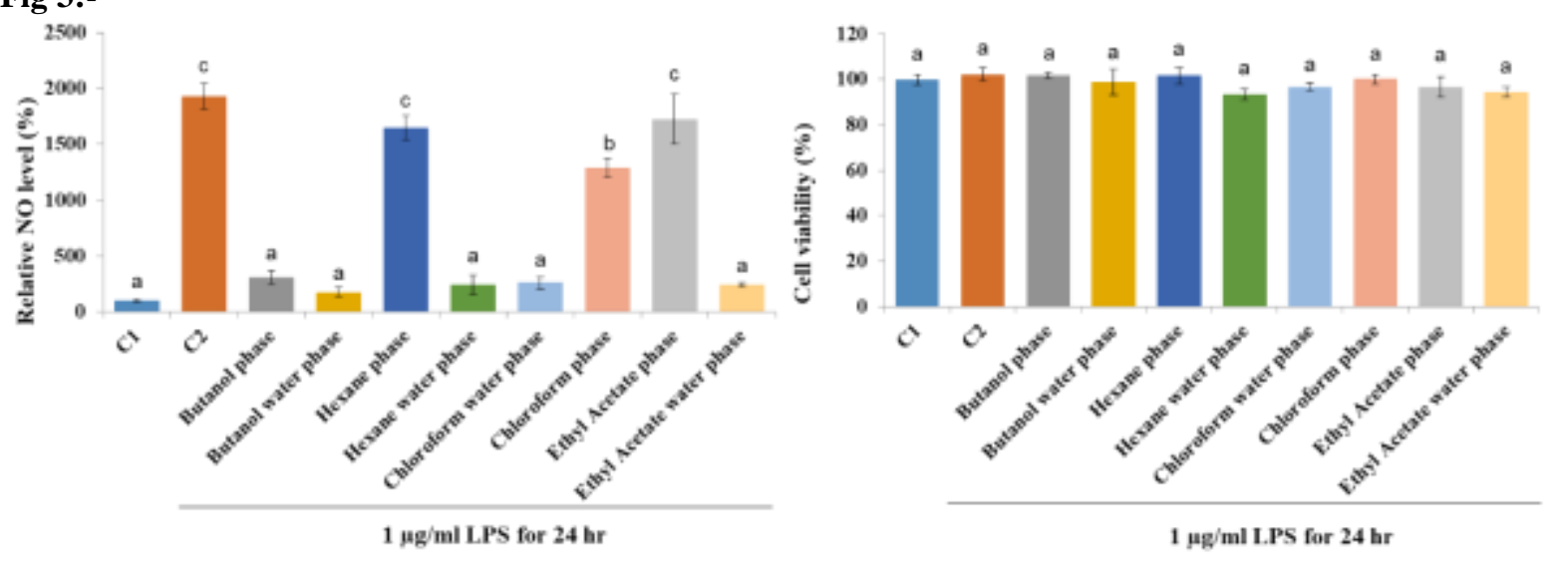


Fig 4:-
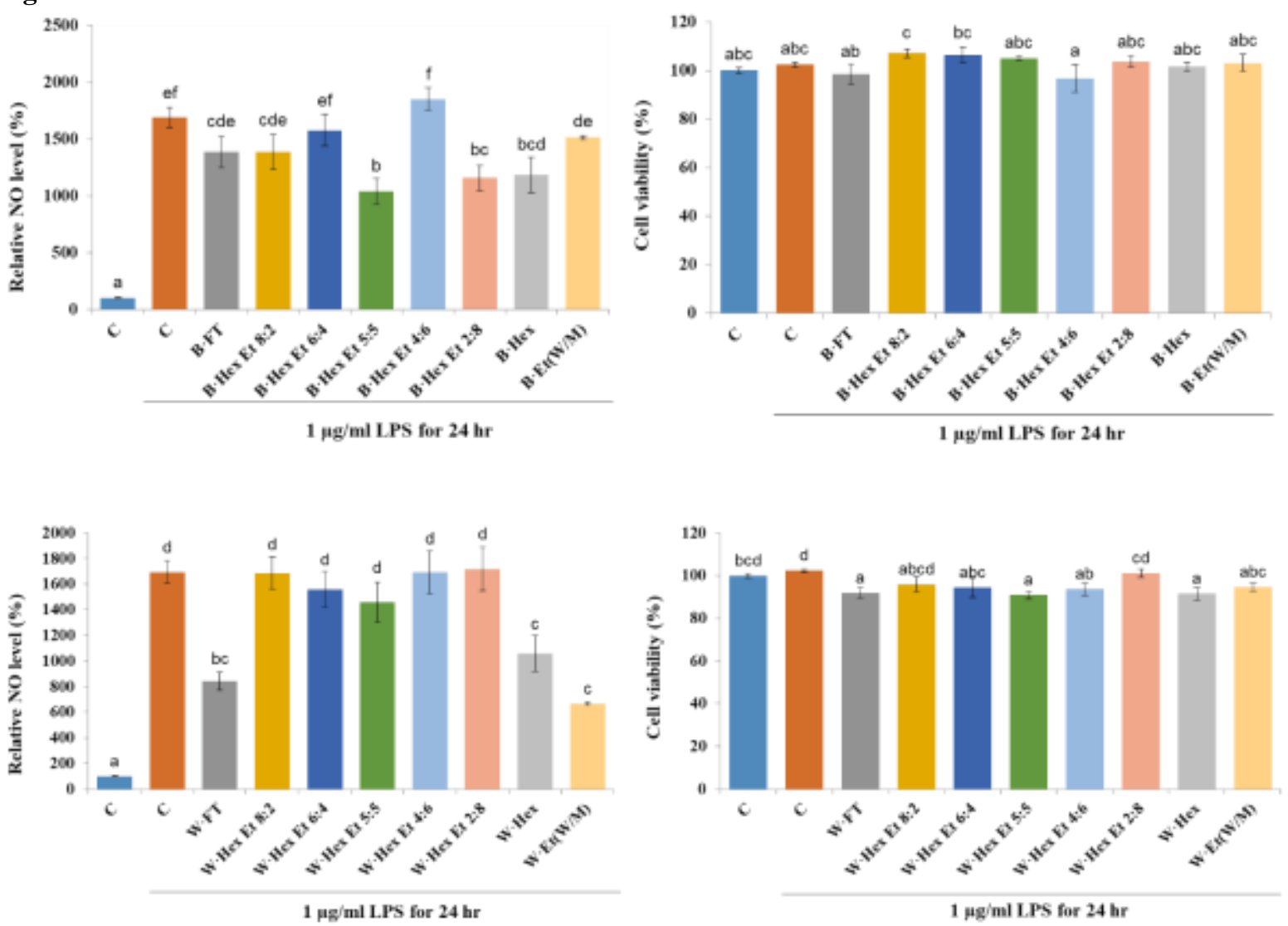

\section{Results and Discussion:-}

Analysis of antiencephalitis and yield by crude extract:-

Antiencephalitic analysis was carried out by 57 kinds extracted from various medicinal herbs. As a result, some of the extracts inhibited cell survival by the extract itself as shown in Fig. 1. The materials to inhibit cell survival are $100 \% \mathrm{MeOH}$ extract of Cimicifugae Rhizoma, 100\% MeOH extract of Rubus coreanus, $100 \% \mathrm{MeOH}$ extract of Panax notoginsengs, $100 \% \mathrm{MeOH}$ extract of Caesalpinia sappan, water extract of C. sappan, and $70 \% \mathrm{EtOH}$ extract of Cinnamomum cassia Presl.

Among the substances that do not inhibit cell survival, the antiinflammatory effect is caused by the hot water extract of L. platyphylla, $70 \%$ EtOH extract of L. platyphylla and water extract of P. notoginseng.

Extraction was carried out by hot water and homogenization to increase the extraction yield of functional materials in L. platyphylla tuber. In the hot water extract, the total solid content was $74.7 \mathrm{~g} / 100 \mathrm{~g}$ dry sample, and the yield was $74.7 \%$ (Table 1). However, the total solid content of the homogenized L. platyphylla tuber was $48.8 \mathrm{~g} / 100 \mathrm{~g}$ dry sample and the yield was $48.8 \%$. Therefore, although it is time - consuming, hot water extraction, a repeated extraction while the original shape of the tuber is maintained, tends to increase the extraction yield rather than the single extraction by fine homogenization.

\section{Methanol fractionation after hot water extract from L. platyphylla tuber;-}

The hot water extract from L. platyphylla tuber inhibits the production of inflammatory mediators such as nitric oxide (NO), interleukin-6 (IL-6), tumor necrosis factor (TNF) and prostaglandin (PG) E2 on RAW 264.7 macrophages cells treated by LPS (Kim et al., 2016). Therefore, in this study, antiencephalitic activity was measured for centrifugal supernatant and pellet according to methanol concentration to analyze functional materials in hot water and homogenized extracts. As shown in Fig. 2, antiencephalitic activity was exhibited from HS50 (homogenized extract and then 50\% methanol supernatant), HS70 (homogenized extract and then $70 \%$ methanol supernatant), HP50 (homogenized extract and then 50\% methanol precipitated pellet), HP70 (homogenized extract 
and then $70 \%$ methanol precipitated pellet), WS50 (hot water extract and then 50\% methanol supernatant), WS70 (hot water extract and then 70\% methanol supernatant), WP30 (homogenized extract and then 30\% methanol precipitated pellet), WP50 (homogenized extract and then 50\% methanol precipitated pellet), WP70 (homogenized extract and then $70 \%$ methanol precipitated pellet). In particular, WP70 showed the strongest activity.

\section{Organic solvent partition:-}

Previous studies have shown that ophiopogonin D and spicatoside A maintain an anti-inflammatory effect (Park et al., 2014). As a result of methanol fractionation in this study, since $70 \%$ methanol precipitate showed the highest activity, this fraction was employed for organic solvent partition. The applied organic solvents for partition were employed by butanol, hexane, chloroform, and ethyl acetate. Each layer from partition was separated and used for analysis of antiencephalitis. The organic solvent layer showed little activity except butanol, whereas all water layers exhibited high activities.

Specifically, since the butanol partition showed activities in both the organic and water layers, additional analysis was carried out using these fraction. In previous studies, ophiopogonin D and spicatoside A from organic solvents are separated into chloroform layers (Park et al., 2014). In this study, however, the activity was observed in both the water and organic solvent layers of butanol. Although this organic solvent extract might contain ophiopogonin D and spicatoside $\mathrm{A}$ in the butanol layer, it is presumed that the other metabolites of bioactive materials are contained in the water layer. The butanol layer was generally less active than the water layer.

\section{Antiencephalic activity of eluents from Sep-Pak R18 catridge:-}

The butanol water and the butanol layers were loaded on a Sep-Pak R18 catridge and then eluted with each eluting solvent (Fig. 4 and 5). In the butanol layer, hexane: ethyl acetate (5: 5) was the most active, and hexane: ethyl acetate (2: 8) and hexane detected activity.

In water layer, the activity was highly found in flow through and ethyl acetate. as mentioned earlier (Park et al., 2014), it is presumed that this result is not related to the activity of ophiopogonin D and spicatoside A, which is associated with antiinflammation. Therefore, we suggest that water layer is involved in antiencephalitis activity by a relatively hydrophilic substance.

\section{Acknowledgement:-}

This work was supported by Priority Research Centers Program through the National Research Foundation of Korea (NRF) funded by the Ministry of Education (2009-0093813).

\section{References:-}

1. Back NI, Cho SJ, Bang MH, Lee IZ, Park CG, Kim MS, Kim KS, Sung JD. 1998. Pesticide environment chemistry. Cytotoxicity of steroid saponins from the tuber of Liriope platyphylla W. T. Agri Chem Biotechnol 41:390-394.

2. Choe SY, Yang KH. 1982. Toxicological studies of antioxidants, butylated hydroxytoluene(BHT) and butylated hydroxyanisole (BHA). J Korean Soc Food Tec 14:283-288.

3. Han DS. 1993. Pharmacognosy. 5th ed. pp.148. Dongmyungsa, Seoul.

4. Hur, J., Lee, P., Moon, E., Kang, I., Kim, S.H., Oh, M.S., Kim, S.Y., 2009. Neurite outgrowth induced by spicatoside A, a steroidal saponin, via the tyrosine kinase A receptor pathway. Eur. J. Pharmacol. 620, 9-15.

5. Kim MJ, Yoo YC, Sung NY, Lee J, Park SR, Shon EJ, Lee BD, Kim MR. 2016. Anti-Inflammatory Effects of Liriope platyphylla in LPS-Stimulated Macrophages and Endotoxemic Mice. Am J Chin Med. 44 (6): 11271143.

6. Kim SD, Ku YS, Lee IZ, Kim ID, Youn KS. 2001. General components and sensory evaluation of hot water extract from Liriopis tuber. J Korean Soc Food Sci Nutr 30:20-24.

7. Kou, J., Sun, Y., Lin, Y., Cheng, Z., Zheng, W., Yu, B., Xu, Q., 2005. Anti-inflammatoryactivities of aqueous extract from Radix Ophiopogon japonicus and its two constituents. Biol. Pharm. Bull. 28, 1234-1238.

8. Park SH, Lee HJ, Ryu J, Son KH, Kwon SY, Lee SK, Kim YS, Hong JH, Seok JH, and Lee CJ. 2014. Effects of ophiopogonin D and spicatoside A derived from Liriope Tuber on secretion and production of mucin from airway epithelial cells. Phytomedicine. 21(2):172-176.

9. Qian, J., Jiang, F., Wang, B., Yu, Y., Zhang, X., Yin, Z., Liu, C., 2010. OphiopogoninD prevents $\mathrm{H}_{2} \mathrm{O}_{2}$-induced injury in primary human umbilical vein endothelialcells. J. Ethnopharmacol. 128, 438-445. 
10. Shin JS. 2002. Saponin composition of Liriope platyphylla and Ophiopogon japonicus. Korean J Crop Sci 47:236-239.

11. Shibata M, Noguchi R, Suzuki M, Iwase H, Soeda K, Niwayama K, Kataoke E, Hamano M. 1971. Pharmacological studies on medicinal plant components. I. On the extracts of Ophiopogon and some folk medicine. Proc Hoshi Pharm 13:66-76. 